Notfall Rettungsmed 2014 · 17:444-444

DOI 10.1007/s10049-014-1844-9

Online publiziert: 25 . Juli 2014

c) Springer-Verlag Berlin Heidelberg 2014

\section{Primäre Patientenzuweisung bei Schädel-Hirn-Trauma (SHT)}

\section{Zum Beitrag}

Esmer E, Steinmetz A, Zeh A et al (2013)

Intrakranielle Blutung bei Polytrauma und

leichtem Schädel-Hirn-Trauma. Notfall

Rettungsmed 16:532-538

\section{Leserbrief}

\section{Richter ${ }^{1,2}$, A. Günther ${ }^{1,2}$, M. Goedecke ${ }^{2}$, T. Gösling ${ }^{3}$ \\ 'Berufsfeuerwehr Stadt Braunschweig \\ ${ }^{2}$ Klinik für Anästhesiologie, \\ Klinikum Braunschweig gGmbH \\ ${ }^{3}$ Klinik für Orthopädie und Unfallchirurgie, \\ Klinikum Braunschweig gGmbH}

Die Arbeit von Esmer et al. zu Patienten mit intrakranieller Blutung bei Polytrauma und leichtem SHT betrachtet die Daten des Traumaregisters unter Aspekten der prähospitalen Versorgung. Ein vielversprechender Ansatz zur Bearbeitung brennender Fragen zur prähospitalen Patientenversorgung und zur Planung von rettungsdienstlichen Versorgungsstrategien.

Die zitierte Arbeit geht von der Annahme aus, dass „... durch Auswahl einer geeigneten Zielklinik mit neurochirurgischer Abteilung... die präklinisch zutreffende Verdachtsdiagnose ... die Prognose des Patienten entscheidend mitbeeinflusst“. Diese Annahme wird nicht belegt. Studien mit relevanten klinischen Endpunkten werden nicht angeführt.

Das Verfahren, Mehrfachverletzte mit SHT primär in ein Zentrum mit neurochirurgischer Versorgung zu transportieren, auch wenn eine Primärversorgung in einem Traumazentrum ohne Neurochirurgie schneller möglich wäre, wird gelegentlich praktiziert. Ausreichende Evidenz für diese Auswahl des primären Ziel- krankenhauses ist uns nicht bekannt. Mit der gültigen Leitlinie zur Polytraumabehandlung [1] ist dieses Verfahren nicht vereinbar. Leitliniengerecht wäre eine Auswahl des primären Zielkrankenhauses nach den Vorgaben von Rettungsdienst und Traumanetzwerk [1].

Wenn etablierte Zuweisungsstrategien nicht befolgt werden, steigt die Gefahr, dass die Priorisierung lebensrettender Behandlung nicht leitliniengerecht erfolgt. Solange unklar ist, ob und ggf. welche Patienten von längeren Transportzeiten zugunsten einer frühen neurochirurgischen Mitbehandlung profitieren würden, halten wir eine Verzögerung der primären Schockraumversorgung für inakzeptabel.

Somit führt die Arbeit von Esmer et al. zu der aus rettungsdienstlicher Sicht relevanten Frage:

Ist das prähospitale Erkennen einer intrakraniellen Blutung entscheidend für das Outcome des Patienten? Falls dieses zutreffen sollte: Welche Konsequenzen ergeben sich aus dieser prähospitalen Diagnosestellung für welche Patienten in den jeweiligen Versorgungsstrukturen?

Mit den Daten des Traumaregisters könnte es möglich sein, mehr Evidenz für die Planung von Zuweisungsstrategien des Rettungsdienstes innerhalb der verschiedenen Traumanetzwerke zu erzeugen. Es sollte möglich sein, die Häufigkeit einer frühen neurochirurgischen Operations- und Interventionspflicht im Rahmen der Polytraumaversorgung festzustellen. Wir hoffen, dass mit der gerade beginnenden Weiterentwicklung und Aktualisierung der S3-Leitlinie Polytrauma [2] hier deutlichere Empfehlungen ausgesprochen werden können.

Bis zum Vorliegen höherer Evidenz oder einer anderen Empfehlung auf Leitlinienniveau sollten Notärzte und Ret- tungsfachpersonal die lokale Zuweisungsstrategie des Rettungsdienstes und der Traumanetzwerke im Interesse ihrer $\mathrm{Pa}$ tienten befolgen.

\section{Korrespondenzadresse}

Dr. A. Günther

Berufsfeuerwehr Stadt Braunschweig

Feuerwehrstraße 1

38114 Braunschweig

\section{Einhaltung ethischer Richtlinien}

Interessenkonflikt. C. Richter, A. Günther, M. Goedecke und T. Gösling geben an, dass kein Interessenkonflikt besteht.

\section{Literatur}

1. Deutsche Gesellschaft für Unfallchirurgie (2011) S3 Leitlinie Polytrauma/Schwerverletzten-Behandlung. http://www.awmf.org/leitlinien/detail/ II/012-019.html

2. Waydhas C (2013) Kontroversen in der Traumatologie. Notfall Rettungsmed 16:512-513 